\title{
Cognitive restructuring and imagery modification in insomnia disorder: a feasibility study
}

\author{
Carolin Schmid ${ }^{1 *}$, Regina Steil ${ }^{1}$ \\ ${ }^{1}$ Institut für Psychologie, Abteilung Klinische Psychologie und Psychotherapie, Goethe Universität Frankfurt
}

\begin{abstract}
Cognitive behavioural therapy for insomnia (CBT-I) is the recommended first-line treatment for insomnia disorder. Although CBT-I shows distinct and robust effects, studies report a stable percentage of non-responders, and many patients do not reach remission. Therefore, new interventions for treatment are needed. Imagery interventions are increasingly popular in cognitive behavioural therapy and might be helpful in insomnia disorder. In this feasibility study, we treated three participants suffering from insomnia disorder with a combination of cognitive and imagery techniques. Participants identified their predominant distressing thoughts and inner images. Methods of cognitive restructuring were used to address automatic thoughts and appraisals. Inner images were evoked in detail and changed into desirable images during an imagery exercise. Participants practised the learned techniques daily for three weeks. One case is described in depth to illustrate the procedure. Sleep onset latency could be reduced by nearly $23 \%$. Subjective insomnia severity and sleep quality could not be changed significantly. Imagery seems to be a promising possibility to enrich CBT-I.

Keywords: Insomnia disorder, cognitive therapy, imagery, adults
\end{abstract}

Insomnia is defined by the DSM-5 (American Psychiatric Association, 2013, p. 362) as a "predominant complaint of dissatisfaction with sleep quantity or quality", especially difficulty initiating or maintaining sleep or earlymorning awakening; insomnia is widespread (prevalence of $5.7 \%$ in the German population; Schlack, Hapke, Maske, Busch, \& Cohrs, 2013) and tends to persist for years (Morin et al., 2009). This disorder has serious effects on patient's lives; for instance, insomnia is predictive of subsequent depression (Baglioni et al., 2011) and is

*Correspondence: carolin.schmidapsych.uni-frankfurt.de Carolin Schmid, Institut für Klinische Psychologie und Psychotherapie, Varrentrappstr. 40-42, 60486 Frankfurt am Main, Germany;

Received: 07 August 2018 Accepted: 08 November 2018

Sleep and Hypnosis

Journal homepage:

www.sleepandhypnosis.org

ISSN: 2458-9101 (Online) associated with suicide risk independent of depression (Drapeau \& Nadorff, 2017; Etindele Sosso, 2017).

Cognitive behavioural therapy is the method of choice for treating patients with chronic insomnia (American Academy of Sleep Medicine, 2017; Qaseem, Kansagara, Forciea, Cooke, \& Denberg, 2016). This treatment first focused on behavioural strategies such as sleep restriction, stimulus control and sleep hygiene (cf. Perlis, 2005), which were subsequently supplemented with cognitive models and strategies (Espie, Broomfield, MacMahon, Macphee, \& Taylor, 2006; Harvey, 2005; Harvey et al., 2014).

These treatments are effective, but most patients experience persistent symptoms (Riemann et al., 2015). Bélanger et al. (2016) reported that a maximum of $80 \%$ of the participants were responders and that $73 \%$ of the participants were in remission in a study investigating CBT-I, and Morin et al. (2009) reported maximum remission values of $60 \%$. Therefore, new approaches are needed. 
Using imagery to change cognitions has become increasingly popular in cognitive behavioural therapy (cf. Arntz, 2012; Hackmann, Bennett-Levy, \& Holmes, 2011; Holmes, Arntz, \& Smucker, 2007) and has proven to be effective for a variety of psychiatric disorders in a metaanalysis (Morina, Lancee, \& Arntz, 2017). Our work group employs a combination of interventions for cognitive restructuring and exercises for imagery modification (CRIM). This procedure was developed to treat the feeling of being contaminated (FBC), which is a widespread phenomenon in PTSD patients, and showed good effects in reducing FBC and general PTSD symptoms (Jung \& Steil, 2013; Steil, Jung, \& Stangier, 2011). Recently, we successfully treated low self-esteem in PTSD patients with the same methodology (Müller-Engelmann \& Steil, 2017). Imagery is already successfully applied in the treatment of nightmare disorder (Imagery Rehearsal Therapy, Krakow \& Zadra, 2006) and showed good effects in a meta-analysis (Hansen, Höfling, KrönerBorowik, Stangier, \& Steil, 2013).

Although insomnia patients report spontaneous visual intrusions (Nelson \& Harvey, 2003), thus far, imagery has only been used as a means of relaxation or distraction from rumination (Morin \& Azrin, 1987). The present study aimed to determine whether imagery is a promising technique for improving insomnia symptoms. We expected the imagery treatment to reduce sleep onset latency and the subjective severity of insomnia and to enhance sleep quality.

\section{MATERIALS AND METHODS}

The study was approved by Frankfurt university clinic's ethical board. This study was not registered in a clinical trials registry due to its preliminary nature. The recruitment started in October 2016, the first treatment session was delivered in December 2016, and the final session was delivered in July 2017.

\section{Participants}

The participants were recruited via a sleep disorder outpatient clinic at the university hospital of Goethe University Frankfurt and a centre for outpatient psychotherapy at Goethe University. We restricted the sample to patients with a predominant complaint of insomnia because we wanted to test the intervention's effect on primary problems falling asleep rather than other diseases causing sleep problems. Patients were eligible if they presented with problems initiating sleep that met the criteria for insomnia disorder according to the DSM-5 (American Psychiatric Association, 2013) that had been present for at least 6 months and a sleep onset latency (SOL) of more than 30 min on 3 or more nights a week. Furthermore, the patients were required to be taking stable medication (psychopharmacologic and sleep medication or other drugs potentially influencing sleep) for the prior three months, be at least 18 years old, and have sufficient knowledge of German.

The exclusion criteria were another somatic or psychiatric disease clearly causing the sleep problems or dominating complaints, a lifetime-diagnosis of bipolar disorder, a psychotic or substance-related disorder, a body mass index lower than 16 , acute suicidality or acute risk of harming others, and mental retardation. Furthermore, the patients were excluded if they were currently receiving another psychological treatment focusing on sleep problems.

All participants provided informed consent (oral and written) for the data collection and publication. Their privacy rights were granted by the following means: Personal data were recorded only for appointments and administrative reasons. Data concerning diagnoses, course of therapy, and measures were recorded with a study code. The digitalization and data analysis were performed using anonymized data, which were aggregated, except for the individual course presented in the case report. All study staff adhered to professional discretion.

\section{Intervention}

All treatments were conducted by the authors at the centre for outpatient psychotherapy. The treatment manual was adapted from the CRIM procedure for PTSD patients (Müller-Engelmann \& Steil, 2017). The intervention consisted of six weekly 50 -min sessions with a three-week training phase before the final session.

See Figure 1 for an overview of the procedure. 
Sessions
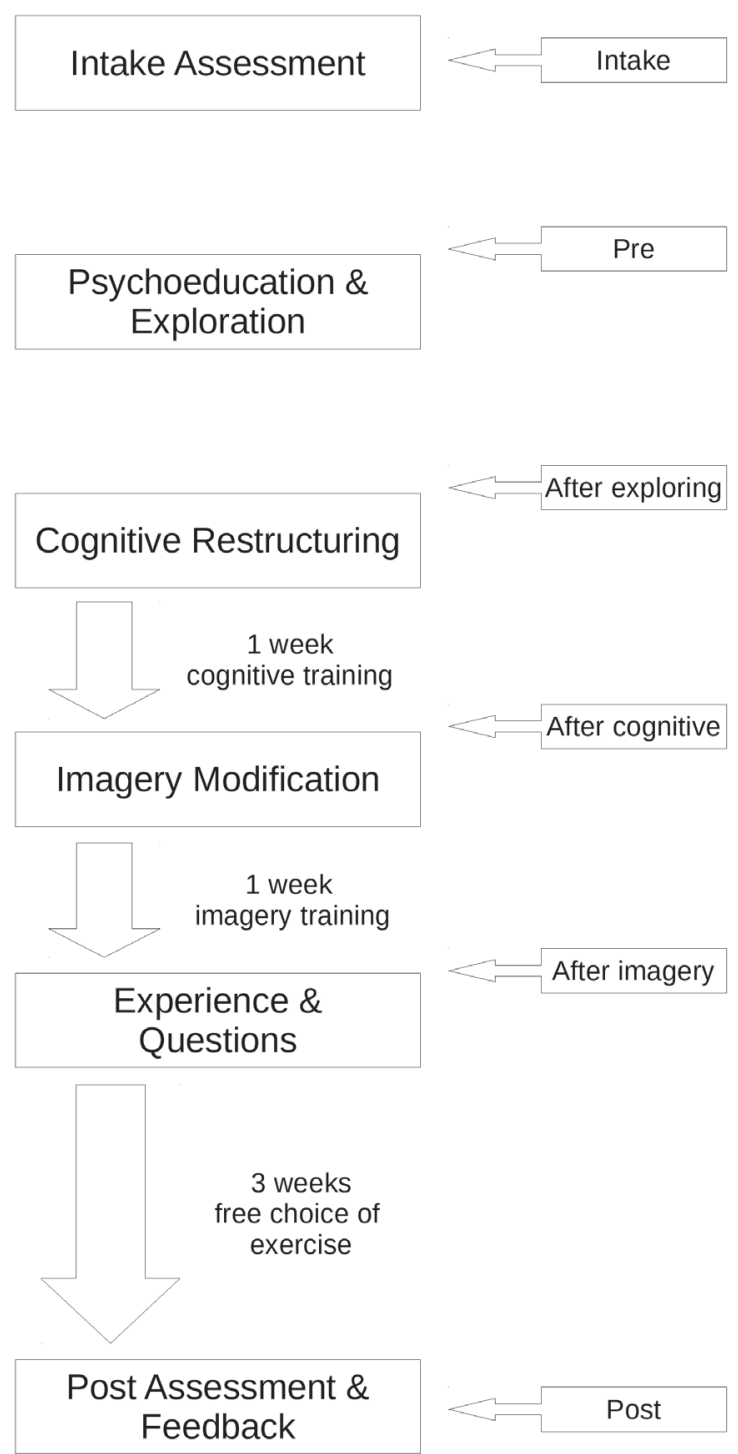

Figure 1. Overview of the Therapy Procedure

The first session was dedicated to establishing the diagnosis and intake assessment.

The second session started with psychoeducation regarding insomnia and the dysfunctional role of cognitions. The participants' individual thoughts and spontaneous inner images were explored and those with the highest personal importance were recorded for future sessions (e.g., "I will never get to sleep again" or "This makes me ill"). Similarly, the participants were asked if they experienced spontaneous imagery when trying to fall asleep. Images of subjective importance (e.g., seeing themselves like in a tight diver's suit, unable to move freely) were recorded.

The third session was dedicated to discussing dysfunctional thoughts by established cognitive restructuring techniques. The participants were asked to recapitulate alternative appraisals at home by writing a plea against their previous convictions and for their alternative views and thoughts. The participants were instructed to rehearse or revise this plea daily until the following session.

During the fourth session, the participants were guided to create an idiosyncratic inner image of their sleep difficulties. The image could pertain to the sleep problem or its consequences and could be concrete or symbolic (e.g., a picture of oneself lying in bed turning over and over or a picture of a floppy balloon lying on the floor to symbolize the feeling in the morning; see Case Report for examples). The pictures were enriched with as many sensory details as possible. Subsequently, the participants developed an alternative image of how they would like to feel or would like the situation to be. The therapists fostered imagination without influencing the content of the imagery.

After the development of both pictures, the participants were guided through an imagination exercise during which they first imagined the dysfunctional image and then changed it step-by-step to the functional alternative. The participants were provided an audio recording of this exercise (10-15 $\mathrm{min}$ ) and asked to rehearse it daily until the following session.

The participants' personal experience and questions regarding the training in daily life were discussed during the fifth session. The typical topics included finding a suitable time and place for practice, handling troubling thoughts and distraction, improving their images, considering new arguments for pleas or asking questions that arose while practicing.

This session was followed by a three-week training phase. The participants were asked to conduct one exercise of their choice per day (rehearsing or revising the plea or imagery exercise). The final session was dedicated to post-intervention assessments and personal feedback. 


\section{Assessments and Measures}

The measures were chosen following the "Recommendations for a Standard Research Assessment of Insomnia" (Buysse, Ancoli-Israel, Edinger, Lichstein, \& Morin, 2006). We deemed the whole recommended set too exhaustive for a small explorative study. Thus, we restricted the measures to three. The participants completed the questionnaires directly after the first session and prior to each subsequent session. In addition, the participants kept a sleep diary throughout the study period.

\section{Diagnosis}

The insomnia diagnosis was evaluated according to the DSM-5 criteria. Other disorders were diagnosed by the structured clinical interview for the DSM-IV-TR, German translation (Wittchen, Zaudig, \& Fydrich, 1997) during the intake session.

\section{Sleep diary}

We used the consensus sleep diary (Carney et al., 2012, German translation by the first author). Each morning, the participants recorded how long it had taken them to fall asleep the night before, and the sleep onset latency (SOL) was calculated as a weekly mean. Test-retest reliabilities between $r=.56$ and $r=.66$ are reported for a similar diary over 22 months (Monk et al., 1994); higher values (between $r=0.84$ and $r=0.93$ ) have been reported for consecutive nights (Smith \& Wegener, 2003). Correlations between sleep diaries and polysomnographic data vary widely; for example, correlations between $r=$ .62 and $r=.98$ were reported for sleep latency in an overview of psychometric findings (Smith \& Wegener, 2003).

\section{Insomnia Severity Index (ISI)}

The ISI (Morin, 1993) consists of seven items pertaining to insomnia symptoms and impact on daily activities and performance. The items are rated on a five-point Likert scale, and the ratings are summed. The questionnaire provides cut-off values for subthreshold insomnia, clinical insomnia (moderate severity) and clinical insomnia (severe). We used the German version published by
Gerber et al. (2016, additional material). The authors report sufficient internal consistency (Cronbach's alpha = $.76 / .77 / .81$ in a series of three studies) and correlations of approximately $r=.5$ with sleep quality (PSQl).

\section{Pittsburgh Sleep Quality Index (PSQI)}

The PSOI (Buysse, Reynolds, Monk, Berman, \& Kupfer, 1989) consists of 25 items. This index is used to obtain typical bed and sleep times, ratings of different sleep quality problems, and a bed partner's rating of breathrelated factors. Higher scores indicate lower quality of sleep.

We used the German version published on the website of the German Society for Sleep Research and Sleep Medicine (Deutsche Gesellschaft für Schlafforschung und Schlafmedizin [DGSM]). For this version, the following psychometric criteria are reported: internal consistency (Cronbach's alpha) $=.85$, test-retest reliability $r=.87$; correlation with sleep diaries $r=.71$ for SOL, and correlations with polysomnographic measures $r=.27$ for sleep time, $r=.28$ for SOL (Backhaus, Junghanns, Broocks, Riemann, \& Hohagen, 2002).

\section{Data Analysis}

We compared the intake assessment of the ISI and PSQI (first session) to the post assessment (final session) and the SOL in the sleep diary of the first trial week (between sessions one and two) to the SOL in the diary of the final week (third week of training before session six). We performed paired-samples t-tests using SPSS Version 22 (IBM Corp., 2013). The one-tailed level of significance was set to .0167 (.05, Bonferroni-corrected for 3 tests). The effect sizes and post hoc power analyses were computed with G*Power (Faul, 2014).

\section{RESULTS}

\section{Participants}

Three participants completed the treatment. Two participants were males, and one participant was female. The mean age was 36 years $(21,43$, and 43$)$. The participants had suffered from insomnia disorder for an average of 26 months $(6,12$, and 60$)$. All participants met 
the diagnostic criteria for insomnia disorder with difficulties initiating sleep. None of the participants had current comorbidities, and one participant previously suffered from panic disorder (F40.0). One participant was a university student, and the other two participants had an academic background. Two participants lived in a partnership, and one participant was single.

\section{Descriptive Data}

The descriptive statistics of the outcome measures of all three participants are shown in Table 1.

\section{Test Results}

We performed paired-samples t-tests to compare the outcome measures before and after treatment. The results are reported in Table 2.

The SOL was the only measure that significantly changed (reduction by $23 \%$, mean difference $=0: 16 \mathrm{~h}$, $t(2)=5.93, p=.027$, two-tailed, corresponding to .0135 one-tailed).

\section{Effect Sizes and Post Hoc Power Analysis}

The effect size of the reduction in SOL is very large $\left(d_{z}\right.$ $=3.67)$. The effect size of the reduction in subjective severity (ISI) was medium $\left(d_{z}=0.42\right)$, and the effect size of the gain in sleep quality (PSQI) was substantial $\left(d_{z}=1.09\right.$; the formula employed by $G^{*}$ Power is reported in Heinrich Heine Universität, 2017, p. 45). The power reached was $(1-\beta)=.99$ for SOL, $(1-\beta)=.13$ for ISI and $(1-$ $\beta)=.36$ for PSQl.

\section{Clinical Significance}

The post assessment values of all measures remained in the clinical range. For SOL, at least 30 min three times per week are suggested as a standard by Buysse et al. (2006). This suggestion is equivalent to approximately 13 min per day, and our participants reported an average SOL of 53 min per day. The average ISI post assessment value (12.75) is in the range of subthreshold insomnia (814). For the German version of the PSQI, a cut-off of 6 is proposed (Backhaus et al., 2002), and the average value of our participants was 10 .

Table 1. Descriptive Values of the Outcome Measures

\begin{tabular}{|c|c|c|c|c|c|}
\hline Measure & $\mathbf{N}$ & Minimum & Maximum & Mean & SD \\
\hline SOL pre & 3 & $0: 24$ & $1: 48$ & 1:09 & $0: 42$ \\
\hline SOL post & 3 & 0:05 & $1: 30$ & $0: 53$ & $0: 43$ \\
\hline ISI intake & 3 & 13 & 18 & 16.00 & 2.65 \\
\hline ISI post & 3 & 8 & 19 & 13.33 & 5.51 \\
\hline PSQI intake & 3 & 9 & 13 & 11.67 & 2.31 \\
\hline PSQI post & 3 & 7 & 13 & 10.00 & 3.00 \\
\hline
\end{tabular}

Note. $S D$ = standard deviation, SOL = Sleep Onset Latency, ISI = Insomnia Severity Index, PSQI = Pittsburgh Sleep Quality Index.

Table 2. Paired-Samples t-Tests

\begin{tabular}{|c|c|c|c|c|c|c|c|c|}
\hline & \multicolumn{5}{|c|}{ Paired Differences } & \multirow[b]{3}{*}{$\mathbf{t}$} & \multirow[b]{3}{*}{ df } & \multirow[b]{3}{*}{ Sig. (2-tailed) } \\
\hline & \multirow[b]{2}{*}{ Mean } & \multirow[b]{2}{*}{ SD } & \multirow[b]{2}{*}{ SEM } & \multicolumn{2}{|c|}{$95 \% \mathrm{Cl}$ of the Difference } & & & \\
\hline & & & & Lower & Upper & & & \\
\hline $\begin{array}{l}\text { SOL pre - } \\
\text { SOL post }\end{array}$ & $0: 16$ & $0: 04$ & $0: 02$ & $0: 04$ & $0: 27$ & 5.93 & 2 & $.027^{\star}$ \\
\hline $\begin{array}{l}\text { ISI intake - } \\
\text { ISI post }\end{array}$ & 2.67 & 6.43 & 3.71 & -13.30 & 18.64 & .72 & 2 & .547 \\
\hline $\begin{array}{l}\text { PSQI intake - } \\
\text { PSQI post }\end{array}$ & 1.67 & 1.53 & .88 & -2.13 & 5.46 & 1.89 & 2 & .199 \\
\hline
\end{tabular}

Note. SD = standard deviation, SEM = standard error of mean, $\mathrm{Cl}=$ confidence interval, SOL = Sleep Onset Latency, ISI = Insomnia Severity Index, PSQI = Pittsburgh Sleep Quality Index, ${ }^{*}=p<.05$. 


\section{DISCUSSION}

We could only confirm our hypothesis regarding SOL. The subjective measures of the insomnia severity and sleep quality did not significantly change. All post assessment values remained in the clinical range.

These effects are weaker than those observed for CRIM in our other studies, which targeted specific symptoms of PTSD, such as feeling contaminated or low self-esteem (Jung \& Steil, 2013; Müller-Engelmann \& Steil, 2017; Steil et al., 2011).

In a meta-analysis of imagery rescripting as an intervention for aversive memories, Morina et al. (2017) observed a large effect size for uncontrolled pre-posteffects on the primary outcome measures (Hedge's $g=$ 1.22 for the studies included, $g=1.06$ considering possible publication bias via the trim and fill procedure). Two of the three effect sizes in this study are even higher (SOL and PSQI), but the explanatory power of our parameters is limited given the small sample size.

\section{Limitations}

Our sample was small, even for a feasibility study. The statistical tests can be regarded as preliminary at best. All participants were Caucasians with a high educational level. The exclusion criteria eliminated patients with other dominant complaints. Thus, our results cannot be generalized to the general population or patients with comorbidities. All participants received cognitive and imagery interventions. Hence, we cannot separate the respective contributions, and we cannot rule out the possibility that cognitive interventions alone or the mere relaxing effect of imagery accounted for the change in SOL. We cannot draw conclusions regarding the stability of the therapy effects because we lack follow-up data. Our intervention consisted of three intervention sessions and five weeks of training. This period is rather short compared to that recommended in established treatment manuals for insomnia; for instance, Perlis (2005) recommends eight sessions, and Harvey (2005) recommends six to ten sessions. More time for elaboration and training on the techniques might have resulted in better effects.

\section{CONCLUSION}

Our feasibility study was an initial test of the usefulness of imagery therapy for participants suffering from insomnia disorder. A combination of imagery and cognitive therapy elements over a five-week training period was able to reduce the mean SOL by $23 \%$ in three pilot participants. The imagery techniques were easily understood and applied by the participants.

Measures of imaging ability (Pearson, Deeprose, Wallace-Hadrill, Burnett Heyes, \& Holmes, 2013) might help identify persons who could especially benefit from these techniques. Future studies involving larger samples, adequate therapy length, follow-up data and a dismantling design might help determine whether the imagery techniques independently contribute to the therapy effects.

\section{CASE REPORT}

\section{Personal Data}

The participant was a 43-year-old female with an academic background. She presented with a 20 -year history of problems falling asleep and met the diagnostic criteria for 5 years.

\section{Images}

Her first image regarding her sleeping problems was generated from the situation of lying in bed and turning over for hours while trying to fall asleep. She depicted an earthworm, brown and shining, curled up and stiff similar to metal. The place was cold and there was a sharp smell. She could feel her breath stopping and pressure on her chest. She changed the image to a colourful flowering meadow in the warm sunshine, with blue skies and the fragrance of flowers. The earthworm relaxed and sprawled, became pink in colour and very long, enjoyed the sunshine and moved freely. The participant experienced a remarked change in emotion and bodily sensation, and she could breathe freely.

The participant worked with a similar image of a wounded deer forced to flee in an attempt to symbolize 
the situation of being awakened and forcing herself to get up after a bad night. The third image she used was symbolic of her feelings during the workday. She imagined herself as a floppy cleaning rag made of holey dirtygreyish cotton, past its peak, threadbare and worn out but spreading out and trying its best to still clean.

\section{Course}

The participant started with an SOL of 1:16 h. She reduced that time to 52 min over the course of therapy but ended with 1:06 h because of a night flight among other factors. Her ISI values oscillated between 13 and 15 , which is approximately the cut-off between

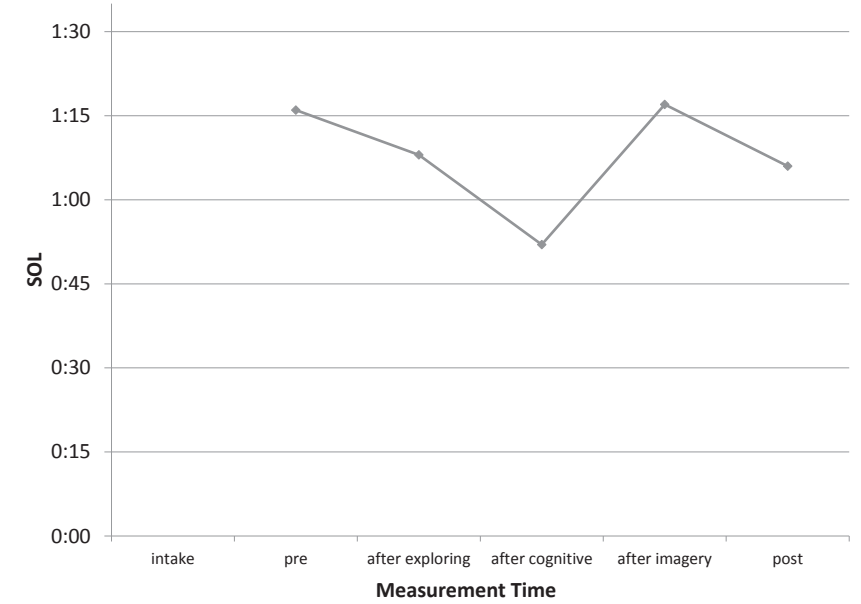

Figure 2. Sleep Onset Latency over the Course of Therapy

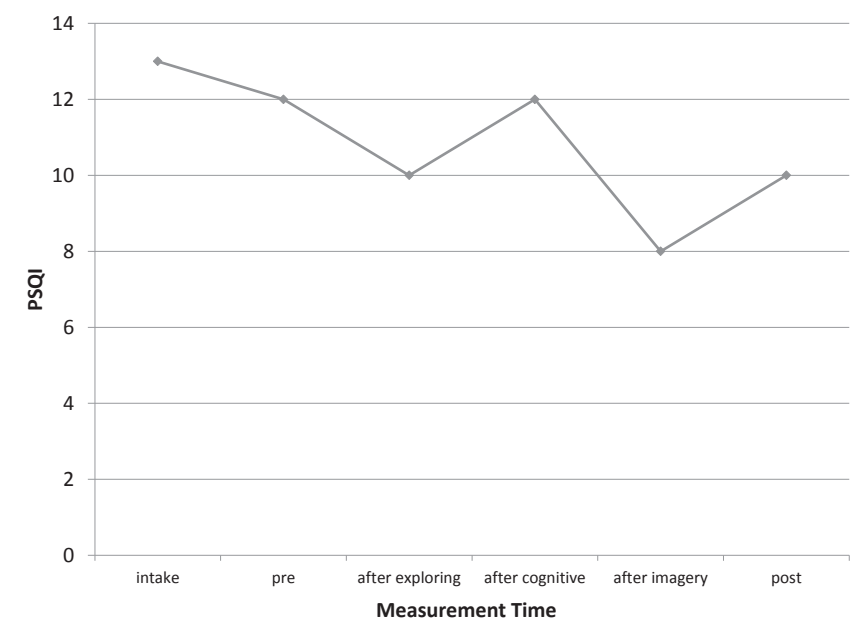

Figure 3. Pittsburgh Sleep Quality Inventory over the Course of Therapy "subthreshold insomnia" and "clinical insomnia (moderate severity)". Her sleep quality slightly improved from a PSOI value of 13 to a value of 10 .

The participant emphasized the insight she had gained into inner processes that she had never thought about before. She reported regarding her sleep from a new perspective.

\section{Therapist's Observations}

The participant spontaneously used pictographic terms to describe how she behaved or felt. She could easily gain access to inner images and supposed that they had been there before treatment, although she had not been aware of them. Images helped her become aware of implicit thoughts and appraisals.

Another participant was less susceptible to the imagery aspect. His imagery remained in the concrete situation of lying in bed and was not accompanied by a marked emotional or bodily reaction.

Acknowledgements: The authors wish to thank the staff of the sleep disorder outpatient clinic at the university hospital of Goethe University Frankfurt (Schlafmedizinische Ambulanz des Universitätsklinikums) for their help in the participant recruitment and Markus Specht, DKD Helios Klinik Wiesbaden, for his advice regarding designing the measurement and recruitment. We thank our participants for providing insight into their imagery. We learned a lot from them. In particular, we thank the participant detailed in the case report for her courage to attempt a new treatment and her consent to the publication of her treatment history.

Funding: This research did not receive any grant from funding agencies in the public, commercial, or not-forprofit sectors.

Informed Consent: Informed consent was obtained from all individual participants included in the study.

Privacy Statement: The patient has read the case report and agreed to its publication.

Conflict of Interest: The authors declare no conflict of interest. 


\section{References}

American Academy of Sleep Medicine. (2017). Practice Guidelines, Psychological and Behavioral Treatment of Insomnia. Retrieved from https://aasm.org/clinical-resources/practice-standards/ practice-guidelines/ Accessed 12 Dec 2017.

American Psychiatric Association. (2013). Diagnostic and statistical manual of mental disorders ( $5^{\text {th }}$ ed.). Washington, DC: American Psychiatric Association.

Arntz, A. (2012). Imagery Rescripting as a Therapeutic Technique: Review of Clinical Trials, Basic Studies, and Research Agenda. Journal of Experimental Psychopathology, 3(2), 189-208. https://doi.org/10.5127/jep.024211

Backhaus, J., Junghanns, K., Broocks, A., Riemann, D., \& Hohagen, F. (2002). Test-retest reliability and validity of the Pittsburgh Sleep Quality Index in primary insomnia. Journal of Psychosomatic Research, 53(3), 737-740. https://doi. org/10.1016/S0022-3999(02)00330-6

Baglioni, C., Battagliese, G., Feige, B., Spiegelhalder, K., Nissen, C., Voderholzer, U.,. . . Riemann, D. (2011). Insomnia as a predictor of depression: A meta-analytic evaluation of longitudinal epidemiological studies. Journal of affective disorders, 135(13), 10-19. https://doi.org/10.1016/j.jad.2011.01.011

Bélanger, L., Harvey, A. G., Fortier-Brochu, É., Beaulieu-Bonneau, S., Eidelman, P., Talbot, L.,. . . Morin, C. M. (2016). Impact of comorbid anxiety and depressive disorders on treatment response to cognitive behavior therapy for insomnia. Journal of Consulting and Clinical Psychology, 84(8), 659-667. https:// doi.org/10.1037/ccp0000084

Buysse, D. J., Ancoli-Israel, S., Edinger, J. D., Lichstein, K. L., \& Morin, C. M. (2006). Recommendations for a standard research assessment of insomnia. Sleep: Journal of Sleep and Sleep Disorders Research, 29(9), 1155-1173. https://doi.org/10.1093/ sleep/29.9.1155

Buysse, D. J., Reynolds, C. F., Monk, T. H., Berman, S. R., \& Kupfer, D. J. (1989). The Pittsburgh Sleep Quality Index: a new instrument for psychiatric practice and research. Psychiatry Research, 28(2), 193-213. https://doi.org/10.1016/01651781(89)90047-4

Carney, C. E., Buysse, D. J., Ancoli-Israel, S., Edinger, J. D., Krystal, A. D., Lichstein, K. L., \& Morin, C. M. (2012). The consensus sleep diary: Standardizing prospective sleep self-monitoring. Sleep: Journal of Sleep and Sleep Disorders Research, 35(2), 287-302. https://doi.org/10.5665/sleep.1642

Deutsche Gesellschaft für Schlafforschung und Schlafmedizin (DGSM). Pittsburgh Schlafqualitätsindex (PSQI) [PDF file]. Retrieved from http://www.dgsm.de/downloads/ fachinformationen/frageboegen/psqi.pdf .

Drapeau, C. W., \& Nadorff, M. R. (2017). Suicidality in sleep disorders: Prevalence, impact, and management strategies. Nature and Science of Sleep, 9, 213-226. https://doi. org/10.2147/NSS.S125597

Espie, C. A., Broomfield, N. M., MacMahon, K. M. A., Macphee, L. M., \& Taylor, L. M. (2006). The attention-intention-effort pathway in the development of psychophysiologic insomnia: A theoretical review. Sleep Medicine Reviews, 10(4), 215-245. https://doi.org/10.1016/j.smrv.2006.03.002

Etindele Sosso, F. A. (2017). Neurocognitive Game between Risk Factors, Sleep and Suicidal Behaviour. Sleep science (Sao Paulo, Brazil), 10(1), 41-46. https://doi.org/10.5935/19840063.20170007

Faul, F. (2014). G*Power. Düsseldorf: Heinrich Heine Universität. Retrieved from http://www.gpower.hhu.de/en.html
Gerber, M., Lang, C., Lemola, S., Colledge, F., Kalak, N., HolsboerTrachsler, E.,. . . Brand, S. (2016). Insomnia Severity Index German Version [DOCX file]. Additional Material to DOI 10.1186/ s12888-016-0876-8. Retrieved from https://static-content. springer.com/esm/art\%3A10.1186\%2Fs12888-016-0876-8/ MediaObjects/12888_2016_876_MOESM1_ESM.docx

Hackmann, A., Bennett-Levy, J., \& Holmes, E. A. (Eds.). (2011). Oxford guide to imagery in cognitive therapy. Oxford guides in cognitive behavioural therapy. Oxford: Oxford University Press.

Hansen, K., Höfling, V., Kröner-Borowik, T., Stangier, U., \& Steil, R. (2013). Efficacy of psychological interventions aiming to reduce chronic nightmares: a meta-analysis. Clinical Psychology Review, 33(1), 146-155. https://doi.org/10.1016/j. cpr.2012.10.012

Harvey, A. G. (2005). A cognitive theory and therapy for chronic insomnia. Journal of Cognitive Psychotherapy, 19(1), 41-59.

Harvey, A. G., Bélanger, L., Talbot, L., Eidelman, P., BeaulieuBonneau, S., Fortier-Brochu, E.,. . . Morin, C. M. (2014). Comparative efficacy of behavior therapy, cognitive therapy, and cognitive behavior therapy for chronic insomnia: a randomized controlled trial. Journal of Consulting and Clinical Psychology, 82(4), 670-683. https://doi.org/10.1037/a0036606

Holmes, E. A., Arntz, A., \& Smucker, M. R. (2007). Imagery rescripting in cognitive behaviour therapy: images, treatment techniques and outcomes. Journal of Behavior Therapy and Experimental Psychiatry, 38(4), 297-305. https://doi. org/10.1016/j.jbtep.2007.10.007

IBM Corp. (2013). IBM SPSS Statistics for Windows. Armonk, NY.

Jung, K., \& Steil, R. (2013). A randomized controlled trial on cognitive restructuring and imagery modification to reduce the feeling of being contaminated in adult survivors of childhood sexual abuse suffering from posttraumatic stress disorder. Psychotherapy and Psychosomatics, 82(4), 213-220. https:// doi.org/10.1159/000348450

Krakow, B., \& Zadra, A. (2006). Clinical management of chronic nightmares: imagery rehearsal therapy. Behavioral Sleep Medicine, 4(1), 45-70. https://doi.org/10.1207/ s15402010bsm0401_4

Monk, T. H., Reynolds, C. F., Kupfer, D. J., Buysse, D. J., Coble, P. A., Hayes, A. J.,. . Ritenour, A. M. (1994). The Pittsburgh sleep diary. Journal of Sleep Research, 3(2), 111-120. https://doi. org/10.1111/j.1365-2869.1994.tb00114.x

Morin, C. M. (1993). Insomnia: Psychological assessment and management. New York, N.Y.: Guilford Press.

Morin, C. M., \& Azrin, N. H. (1987). Stimulus control and imagery training in treating sleep-maintenance insomnia. Journal of Consulting and Clinical Psychology, 55(2), 260.

Morin, C. M., Bélanger, L., LeBlanc, M., Ivers, H., Savard, J., Espie, C. A.,. . Grégoire, J.-P. (2009). The natural history of insomnia: a population-based 3-year longitudinal study. Archives of Internal Medicine, 169(5), 447-453. https://doi.org/10.1001/ archinternmed.2008.610

Morina, N., Lancee, J., \& Arntz, A. (2017). Imagery rescripting as a clinical intervention for aversive memories: A meta-analysis. Journal of Behavior Therapy and Experimental Psychiatry, 55, 6-15. https://doi.org/10.1016/j.jbtep.2016.11.003

Müller-Engelmann, M., \& Steil, R. (2017). Cognitive restructuring and imagery modification for posttraumatic stress disorder (CRIM-PTSD): A pilot study. Journal of Behavior Therapy and Experimental Psychiatry, 54, 44-50. https://doi.org/10.1016/j. jbtep.2016.06.004 
Nelson, J., \& Harvey, A. G. (2003). An exploration of pre®sleep cognitive activity in insomnia: Imagery and verbal thought. British Journal of Clinical Psychology, 42(3), 271-288. https:// doi.org/10.1348/01446650360703384

Pearson, D. G., Deeprose, C., Wallace-Hadrill, S. M.A., Burnett Heyes, S., \& Holmes, E. A. (2013). Assessing mental imagery in clinical psychology: a review of imagery measures and a guiding framework. Clinical Psychology Review, 33(1), 1-23. https://doi.org/10.1016/j.cpr.2012.09.001

Perlis, M. L. (2005). Cognitive behavioral treatment of insomnia: A session-by-session guide. New York, N.Y.: Springer.

Qaseem, A., Kansagara, D., Forciea, M. A., Cooke, M., \& Denberg, T. D. (2016). Management of Chronic Insomnia Disorder in Adults: A Clinical Practice Guideline From the American College of Physicians. Annals of Internal Medicine, 165(2), 125133. https://doi.org/10.7326/M15-2175

Riemann, D., Nissen, C., Palagini, L., Otte, A., Perlis, M. L., \& Spiegelhalder, K. (2015). The neurobiology, investigation, and treatment of chronic insomnia. The Lancet Neurology, 14(5), 547-558. https://doi.org/10.1016/S1474-4422(15)00021-6
Schlack, R., Hapke, U., Maske, U., Busch, M., \& Cohrs, S. (2013). Frequency and distribution of sleep problems and insomnia in the adult population in Germany: results of the German Health Interview and Examination Survey for Adults (DEGS1) [Häufigkeit und Verteilung von Schlafproblemen und Insomnie in der deutschen Erwachsenenbevölkerung: Ergebnisse der Studie zur Gesundheit Erwachsener in Deutschland (DEGS1)] Bundesgesundheitsblatt, Gesundheitsforschung, Gesundheitsschutz, 56(5-6), 740-748. https://doi.org/10.1007/ s00103-013-1689-2

Smith, M. T., \& Wegener, S. T. (2003). Measures of sleep: The Insomnia Severity Index, Medical Outcomes Study (MOS) Sleep Scale, Pittsburgh Sleep Diary (PSD), and Pittsburgh Sleep Quality Index (PSQI). Arthritis \& Rheumatism, 49(5), 184-196. https://doi.org/10.1002/art.11409

Steil, R., Jung, K., \& Stangier, U. (2011). Efficacy of a two-session program of cognitive restructuring and imagery modification to reduce the feeling of being contaminated in adult survivors of childhood sexual abuse: a pilot study. Journal of Behavior Therapy and Experimental Psychiatry, 42(3), 325-329. https:// doi.org/10.1016/j.jbtep.2011.01.008

Wittchen, H.-U., Zaudig, M., \& Fydrich, T. (1997). Skid. Strukturiertes klinisches Interview für DSM-IV. Achse I und II. Handanweisung. 